\title{
Film-Forming Systems in Topically Administered Pharmaceutical Formulations
}

\section{Flora Ferreira Duarte de Oliveira*, Livia Rodrigues de Menezes, Maria Inês Bruno Tavares}

Eloisa Mano Macromolecules Institute, Federal University of Rio de Janeiro, CT, Bloco J, Cidade Universitária, Ilha do Fundão, Rio de Janeiro, Brazil

Email: ^florafdoliveira@ima.ufrj.br

How to cite this paper: de Oliveira, F.F.D. de Menezes, L.R. and Tavares, M.I.B. (2020) Film-Forming Systems in Topically Administered Pharmaceutical Formulations. $\mathrm{Ma}$ terials Sciences and Applications, 11, 576-590. https://doi.org/10.4236/msa.2020.118038

Received: June 15, 2020

Accepted: August 11, 2020

Published: August 14, 2020

Copyright (c) 2020 by author(s) and Scientific Research Publishing Inc. This work is licensed under the Creative Commons Attribution International License (CC BY 4.0).

http://creativecommons.org/licenses/by/4.0/

(c) (i) Open Access

\begin{abstract}
The skin is the most extensive and outermost organ in the body and can be greatly exploited both from the point of view of alternative routes of systemic drug delivery and treatment of dermatological diseases. Because of its main function as a barrier against harmful external agents, it also becomes a barrier to drug administration, but there are strategies to reduce this limitation of this promising route of administration. The development of polymer-based film-forming formulations is extensively studied for this purpose, since the formation of a film on the skin increases the contact time of the drug, for this being characterized as a controlled release reservoir system. There are a multitude of possible polymers to compose these formulations and their choice must be made according to the purpose of each application. This work, therefore, aims to study the state of the art of film forming systems for topical application of pharmaceutical formulations.
\end{abstract}

\section{Keywords}

Film-Forming Systems, Polymeric Formulations, Topical Drug Delivery

\section{Introduction}

Pharmaceutical research faces the major challenge of developing new technologies to give formulations different characteristics that overcome the therapeutic limitations of traditional dosage forms, including adjustable release profiles, flexibility of use, ability to carry more than one active ingredient, improve patient availability and compliance [1].

Whether it is for topical application of dermatological dysfunctions or for transdermal application of systemic action, the skin is an increasingly explored route of administration, as it has no gastrointestinal side effects, avoids the me- 
tabolization of active ingredients by first pass effect, and is of easy application in individuals with swallowing difficulties, such as the elderly and children, for example [1]. However, the skin's main function is to be a barrier against external agents, so, even though it is a promising route of administration, it is not so easily passable [2] [3].

Conventional topical dosage forms, such as cream, gels and ointments, are called semisolids. These usually have an unattractive sensory appearance and do not guarantee prolonged skin contact with the drug, requiring repeated applications throughout the day and may therefore cause less patient adherence to treatment. Therefore, the development of formulations that overcome this technological barrier may be crucial for improving the treatment of dermatological diseases [4] [5].

In this context, polymeric film-forming systems are being explored both in the treatment of dermatological diseases and in cosmetic products as potential formulations to reduce the inconveniences of topical application [4].

Given the above, the main objective of review will be to elucidate the anatomophysiological characteristics of the skin and to conduct a survey of the state of the art of film forming systems in the topical release of pharmaceutical agents.

\section{Skin}

\subsection{Skin Anatomophysiology}

The skin is the most accessible organ of the body, in an adult its surface measures approximately $2 \mathrm{~m}^{2}$ and is what separates the external environment from the interior of the body and performs functions such as: protective barrier against mechanical and chemical actions, pathogen invasion and ultraviolet radiation (UV); water loss barrier, temperature regulation, skin peeling detoxification system, primary defense system, also being a sensory organ and defining appearance. The epidermis is the uppermost layer of the skin and the cell types present in it are keratinocytes, melanocytes, Langerhans cells, and Mekel cells [6] [7] [8].

Keratinocytes are the main components of the epithelial barrier. Basal layer cells are mitotically active and adhere to the basement membrane, which separates the dermis from the epidermis. These multiply and change their morphology, shape and polarity as they move to the uppermost layer of the epidermis. After the basal layer comes to the spinous layer, then to granular and lastly the stratum corneum. When cells reach this last layer, they enter the cell death process, damaging their organelles and their nucleus, ceasing all their metabolic and transcriptional activity and their matrix is filled with dense keratin filaments, forming the skin layer that is in contact with the cell environment, whose component cells are called corneocytes [6].

The melanocytes are distributed in the basal layer and its function is to produce melanin and transport it to the keratinocytes, being responsible for the skin tone. Melanin has a protein structure and is found as a pigment within melano- 
cytes. It is the main agent against harmful effects induced by UV rays [6] [9].

Langerhans cells are responsible for recognizing and eliminating antigens that cross the epidermal barrier. They are usually located in the spinous layer of the epidermis. Mekel cells are located in the basal layer and are mechanoreceptors. The dermis is located below the epidermis and they interact, which forms the structure of the skin. It can be subdivided into 3 layers: papillary, sub-papillary and reticular layer. It is composed of cells, nerves, blood vessels and epidermal appendages, such as the sebaceous and sweat glands. Most of it is composed of amorphous connective tissue and fibrous and filamentous components, which form the extracellular matrix (ECM). The papillary and sub-papillary layers are flexible, have thin collagen fibers, elastic fibers and are rich in water. The reticular layer is stiffer because the collagen fibers are thicker and connect to each other by elastic fibers, forming a more complex network [6] [10].

The dermis gives the skin structural strength and robustness, assisting in functions such as mechanical protection, sensory stimulation, and thermal regulation. The main cellular constituent of the dermis is fibroblasts [6].

Fibroblasts are responsible for producing the dermis extracellular matrix (ECM) components, which are the structures that confer several key characteristics for skin functions, namely collagen fibers, elastic and proteoglycan fibers, and glycoproteins [11].

Collagen fibers account for $70 \%$ of the dry mass of the skin and are responsible for imparting tensile strength to the skin through its excellent mechanical strength characteristics. Among the different types of collagen fibers in the dermis, collagen I and II are the most abundant. Elastic fibers are responsible for the skin's ability to deform and then retreat to its previous state, a property called resilience [11] [12].

Finally, glycosaminoglycans have the function of maintaining skin hydration, as they are polysaccharide chains that are capable of binding to a high volume of water. The main component of this class is hyaluronic acid [11] [12].

The last layer of the skin is the hypodermis. It is the subcutaneous layer, composed of adipocids (fat cells) that assist in thermoregulation, storage, energy supply and mechanical protection functions [6].

\subsection{Skin as A Road of Pharmaceutical Administration}

Topical application refers to any application on any surface, whether on the skin or mucous membranes (vaginal, nasal, ocular). The skin as a route of administration offers several advantages over the others, but because it is the largest and most external organ of the body, its function is to give our body protection against external agents. With active ingredients it is no different, the skin also plays its barrier role when it comes to the absorption of drugs. Normally, reduced quantities of the active ingredient become available for action and the remainder is lost on the surface, not being able to permeate the skin or even the vehicle itself preventing the diffusion of the drug to the site of action [3] [13] 
[14].

As there is such difficulty in overcoming the epidermal barrier, it is often necessary for semi-solid formulations to be applied several times a day to achieve the therapeutic goal, since the substantivity of a topical formulation is related to its permanence in the skin and its resistance to removal by washing and by friction. In addition, these conventional formulations generally lack in sensory attractiveness, which may cause the patient's lack of motivation to reapply the product. All of this may be a reason for poor adherence to treatment [2] [4] [13].

The development of topical formulations that can stay in contact with the skin longer and, consequently, offer a reduction in the need for reapplication is of great dermatological interest [4].

Polymeric film forming systems (FFS) are very promising formulations in the dermatological field, because they overcome the limitations mentioned above. These systems are produced from a solution or emulsion that have in their composition polymers that form films upon application, and these become a reservoir system of the active principle on the skin, thus increasing the time of contact of the drug with the administration pathway [3].

These systems have been studied for transdermal administration of steroid hormones, analgesics, development of mucoadhesive systems for gastric retention, and as an alternative to patching, aiming to improve drug safety and efficacy. In the area of dermatological diseases there are also various applications such as antifungal administration, treatment of burns, wounds, and also have the role in the prevention of transepidermal water loss, thus assisting in skin hydration and other applications in skin care products [4] [15] [16] [17] [18] [19].

\section{Film Forming Systems}

\subsection{Important Parameters in Film Formation}

The process of forming a film appears to be simple, as it consists of spreading a polymer solution over a surface followed by evaporation of the solvent, which results in the formation of the polymer solid film. However, there are some parameters of this process that should be highlighted in order to better understand its step-by-step and to develop smarter systems. They are solution viscosity, polymer solubility in the chosen solvent, polymer chain molar mass and solvent evaporation rate [20].

As the solvent evaporates, the polymer chains interpenetrate when at a gel state and eventually solidify into film upon complete evaporation of the solvent. This phase of interpenetration of the chains is dependent on the viscosity of the solution, which is a property that indicates the hydrodynamic interaction between polymer and solvent. As the concentration of the polymer increases, the viscosity of the solution also increases. Viscosity also depends on the molar mass of the polymer, which, when higher, results in higher viscosities at a given temperature [20] [21].

The solubility parameter is regarded as a qualitative measure of the extent of 
polymer solvation. A greater end-to-end distance of the structure in the polymer chains generally improves the adhesion of the resulting film to the surface where the solution is being applied to, since a larger portion of the polymer is exposed to interact with the surface in question [20]. With the loss of the volatile component of the vehicle, the concentration of the drug to be released also increases, reaching a state of supersaturation on the skin surface. Supersaturation results in improved drug flow by increasing the thermodynamic activity of the formulation without affecting the skin barrier. However, supersaturated systems are unstable and, if they culminate in drug precipitation, all advantages of this type of system are lost [8] [22].

\subsection{Appeal for New Formulation Technologies - Polymers}

Polymers can be extremely versatile components when used in traditional formulations that break new ground for different designs and promise to improve their performance by presenting functions such as capsule binding agents, tablet film coating agents and emulsion and viscosity enhancers. Some of the polymers are administered together with bioactive drug molecules. In addition, polymers can provide more modern systems involving controlled drug release, improved bioavailability and minimization of side effects in different ways, such as [17] [23] [24]:

1) Small size polymer particles that can promote optimal drug release to the site of action;

2) Polymeric systems with aggregated drugs, since they offer controlled release, avoid cases of overdose and side effects and nevertheless release the effective dose;

3) Biodegradable polymers are broken down into biologically compatible molecules and can be metabolized/secreted naturally;

4) Reservoir systems may increase the solubility of certain drugs;

5) They are more easily moldable property systems to meet certain therapeutic treatment needs, such as different molar masses, ligand insertion, etc.

In the context of topical formulations, polymeric film forming systems (FFS) created in situ after administration are a more efficient topical formulation alternative that can promote more patient compliance as it provides the required dose of drug and allows less frequent administration. The rationale involved in this type of formulation is the formation of a film on the skin through the evaporation of the solvent that serves as a drug reservoir system and/or an agent that slows its release, promoting greater control of the active principle contained in its molecular form [4] [5].

One of the advantages of FFSs is that they have more cosmetic/sensory attractiveness attributes than traditional semi-solid formulations because they are fast drying, less greasy and almost invisible. In addition, since it has prolonged skin contact and drug release occurs over a prolonged period, it is necessary that the formed film has sufficient flexibility to adapt to application site movements, high 
substantivity (resistance to sweating, washing, clothing) and strong adhesion to the skin [2] [4].

FFSs are generally classified according to their affinity for water and their substantivity and are divided into 2 categories: hydrophilic or lipophilic [4] [5].

- Hydrophilic: have low substantivity and water resistance. Usually used as skin drug reservoir systems.

- Lipophilic: have greater substantivity and water resistance. Not only do they form reservoir systems on the skin, but they can also form within the skin, for its hydrophobic characteristic is similar to the skin.

One of the critical points of this type of formulation is the immediate evaporation of volatile components, which may lead to the drug supersaturation and, consequently, its crystallization, as the environment becomes thermodynamically unfavorable to its molecular state. This can be controlled by the addition of nucleation inhibiting excipients [13].

In addition, the control of the release profile should be studied, since the chemical nature of the drug and the polymer, as well as their concentrations and the use of other excipients as plasticizers for increased release, directly influence the therapeutic effect [1] [4] [13].

Although the technology of film-forming materials is an innovative and promising approach in the context of controlled drug release, there are still few studies addressing their in vivo use, and further study of the relationship between polymeric matrices and properties is still needed. Materials obtained for the desired biomedical applications can be used either as a wound protection device or as a drug release retardant.

The polymeric matrices used in these formulations are quite varied. Biopolymers, polymers of natural origin, such as alginate, cellulose derivatives, Arabic gum, starch, etc., are examples of polysaccharides widely used in the market. However, they have the disadvantage of variations in quality and lack of safe supply, as it depends on a natural source to be extracted [25].

Synthetic polymers are also well exploited in this type of formulation. Eudragit $\mathrm{R}$ is an acrylate copolymer that is widely used by the pharmaceutical industry as coating agents and may also be applied as film forming agents in FFS [2].

\subsection{Discussion on Film Forming Formulations}

The topic, film-forming systems, has been much discussed in the last 5 years. From 2013 to 2015, although it appeared in several publications, there was a drop in the total amount of studies. However, in 2017 this subject returned to the agenda and again the number of publications on these systems increased, as shown in the chart below.

In order to evaluate how these formulations are prepared in the pharmaceutical field, some studies have been selected focusing on the development of different film forming systems for the most diverse applications in topical formulations, which are proof that these systems go far beyond traditional polymeric 
solution, making it necessary to perform the most varied characterization tests depending on the objective of each study.

The work of Garvie-Cook et al. (2015) presented the evaluation of the effect caused by the addition of a lipid component (medium chain triglyceride - MCT) in the formulation of both hydrophilic (Klucel ${ }^{\circledR}$ LF - hydroxypropylcellulose) and hydrophobic polymers (Eudragit ${ }^{\circledR}$ RS - Acrylate/Ammonium Methacrylate Copolymer and Dermacryl ${ }^{\circledR} 79$ - Acrylate/Octylacrylamide Copolymer). The drug used to evaluate the effect on system release was Betamethasone, a hydrophobic corticosteroid. The developed systems were polymer solutions containing or not MCT or plasticizer, being created from the dissolution of the polymers in absolute ethanol and later addition of the drug. The formulations developed are summarized in "Table 1 " [13].

The results of the release tests vary with the polymer variations chosen and the addition of other components in the formulation (MCT and plasticizer).

The results were performed in an ex vivo pig ear model for the evaluation of the skin uptake profile. The release performances of a single ointment and systems with Eudragit ${ }^{\circledR}$ and Klucel ${ }^{\circledR}$ were compared. The difference in Eudragit ${ }^{\circledR}$ was only due to the difference in drug retention in the stratum corneum, while in the deeper layers of the skin the distribution was similar. Since the absorption

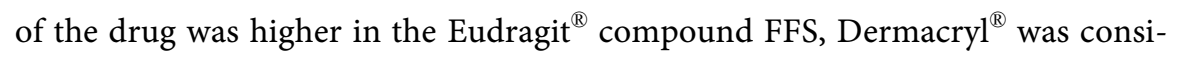
dered to have an even greater hydrophobic character for comparison of results and it was possible to make a relationship between the increase of the absorption of the drug through the skin and the increase of the lipophilicity of the polymer. This happens because hydrophobic components are capable of creating drug interactions that make them a better reservoir in both the polymeric film on the surface and within the stratum corneum, allowing release to occur over a longer period. This also applies to the incorporation of MCT, which increased the hydrophobic load of the formulation and made a significant increase on the drug delivery after 6 - 8 hours of application [13].

Another study that uses polymers and the drug in common with the previous one is that of Frederiksen; Guy; Petersson (2015), whose goal is to create prolonged release formulations using the skin as a route of administration. Firstly, the effect of polymeric film formation was evaluated, and the polymers used were: hydroxypropylcellulose (Klucel $\left.{ }^{\circledR} \mathrm{LF}\right)$; hydrophobic copolymers: polymethacrylate

Table 1. Composition of the film forming system formulations (\% w/w), all with $1.2 \%$ w/w Betamethasone [13].

\begin{tabular}{|c|c|c|c|c|c|c|c|c|}
\hline \multirow{2}{*}{\multicolumn{2}{|c|}{$\begin{array}{c}\text { Formulation Components } \\
\text { Polymer }\end{array}$}} & \multicolumn{3}{|c|}{ Klucel $^{\circledR}$ LF } & \multicolumn{3}{|c|}{ Eudragit $^{\circledR}$ RS } & \multirow{2}{*}{$\frac{\text { Dermacryl }^{\circledR} 79}{10.0}$} \\
\hline & & 5.0 & 5.0 & 5.0 & 15.0 & 15.0 & 15.0 & \\
\hline \multirow{2}{*}{ Plastifier/Lipid } & TEC & - & 1.0 & & - & 3,0 & - & - \\
\hline & MCT & & - & 1.0 & - & - & 3.0 & - \\
\hline \multirow{2}{*}{ Solvent } & Ethanol & 95 & 94 & 94 & 80 & 77 & 77 & 90 \\
\hline & Water & - & - & - & 5.0 & 5.0 & 5.0 & - \\
\hline
\end{tabular}


(Eudragit ${ }^{\circledR}$ NE - Poly(ethyl acrylate-co-methyl methacrylate and Eudragit ${ }^{\circledR}$ RS) and polyacrylate copolymer (Dermacryl ${ }^{\circledR} 79$ ). Then, the impact of the incorporation of plasticizers (triethyl citrate, tributyl and dibutyl sebacate) was analyzed. The formulations are summarized in "Table 2" [4].

In this study also polymer solutions were developed in absolute ethanol, with and without plasticizers (20\% w/w in relation to pure polymer) and with Betamethasone (1.2\% of polymer). The final polymers studied were Klucel ${ }^{\circledR}$, Eudragit (NE and RS) and Dermacryl ${ }^{\circledR}$ at the concentrations that had the best performance in relation to fast drying, translucency and no sticky effect.

Although the methodology used for the development of these solutions is similar to the one from the previous study, new questions were addressed regarding the critical points of formulation for topical application.

Firstly, the addition of plasticizers was evaluated, which did not result in any visual effect on the structural and flexibility characteristics of the obtained films, but the Klucel $^{\circledR}$ formulation became foggier with the addition of more hydrophobic plasticizers (TBC and DBS). In the evaluation by differential scanning calorimetry, it was observed that in all cases, the glass transition temperature of the polymers was reduced with the presence of plasticizers, this was an important factor to be analyzed, since for the films to have an appropriate flexibility on the skin surface, the material $\mathrm{Tg}$ must be less than the skin temperature (approximately $32^{\circ} \mathrm{C}$ ). This parameter was especially important for Eudragit ${ }^{\circledR} \mathrm{RS}$ formulations, which resulted in $\mathrm{Tg}$ of $65^{\circ} \mathrm{C}$, making it necessary to use FFS plasticizers in compositions with this polymer.

Still regarding the role of plasticizers in the studied FFS, all of them increased the release of the drug. This may be justified by the greater mobility of the polymer chains and consequent greater free volume in the formed films, which facilitates the diffusivity of the drug by the film and thus increases its release to the skin.

In relation to the characteristic of polymers, the one that had the highest drug release from the formed film was Klucel ${ }^{\circledR}$. The authors justified this result by its hydrophilicity and the low solubility of Betamethasone in this polymeric matrix, which causes a greater tendency for the drug to escape from the film in question.

Table 2. SFF Formulation Composition (\%p/p) [4].

\begin{tabular}{|c|c|c|c|c|c|c|c|c|c|c|c|}
\hline \multirow{2}{*}{\multicolumn{2}{|c|}{$\begin{array}{c}\text { Formulation Components } \\
\text { Polymer }\end{array}$}} & \multirow{2}{*}{$\frac{\text { Dermacryl }^{\circledR}}{10}$} & \multirow{2}{*}{$\frac{\text { Eudragit }^{\circledR} \mathrm{NE}}{7.5}$} & \multicolumn{4}{|c|}{ Eudragit $^{\circledR}$ RS } & \multicolumn{4}{|c|}{ Klucel $^{\circledR}$} \\
\hline & & & & 15 & 15 & 15 & 15 & 5 & 5 & 5 & 5 \\
\hline \multirow[t]{3}{*}{ Plastifier } & DBS & - & - & & 3 & - & - & - & 1 & - & - \\
\hline & TBC & - & - & - & - & 3 & - & - & - & 1 & - \\
\hline & TEC & - & - & - & - & - & 3 & - & - & & 1 \\
\hline \multirow[t]{2}{*}{ Solvent } & Etanol & 90 & 81.3 & 80 & 77 & 77 & 77 & 95 & 94 & 94 & 94 \\
\hline & Água & - & 11.2 & 5 & 5 & 5 & 5 & - & - & - & - \\
\hline
\end{tabular}

Adapted of FREDERIKSEN; GUY; PETERSON, 2015. 
In addition, the hydrophilic character of Klucel $^{\circledR}$ promotes water absorption through the film over time, which makes the environment even less favorable for the hydrophobic drug, further increasing its release. In contrast, the hydrophobic polymers have the slowest release profile and an initial burst was observed, which shows less control of the polymeric film over drug release.

Film forming solutions are not the only ones available and applicable in the pharmaceutical field. In the study by Tongnuanchan et al. (2015), the researchers prepared a film-forming emulsion. To obtain this type of formulation, a film-forming solution was first prepared from gelatin powder obtained from tilapia skin, a species of fish, which was dissolved in water under heating and then glycerol was added as plasticizer. To this FFS was added the mixture of palm oil (emulsion oil phase) and lecithin (surfactant) at different concentrations, under intense stirring in homogenizer for a few minutes [26].

Unlike film-forming solutions, it has been observed that the addition of oil makes the formulation less translucent. The higher the oil concentration, the less translucent the film formed.

In addition, it was evidenced that increasing the oil concentration in the formulation also had consequences on film thickness, as higher palm oil concentrations resulted in thicker films. This is due to the simple increase of the solid component of the formulation in the form of droplets which make it difficult to package the polymer chains, generating thicker films as the oil concentration increases.

FFS has also been widely studied in tissue repair and wound and burn treatment, as they offer advantages over their application in large areas and drug release profiles, mainly for their ability to prevent body fluid loss and protection against external contamination, among others [17]. In order to create an Aloe vera formulation and a vitamin $\mathrm{E}$ acetate derivative for wound care, different concentrations of the formulation polymers (polyvinyl alcohol - PVA and sodium alginate) were studied, found in "Table 3", as well as their interference with the properties and characteristics of the formed films in "Table 4".

As a result, all films showed apparent adequate flexibility and strength as they were easily manipulated during the study. In addition, all were completely solubilized

Table 3. Concentration (\%p/p) of formulation components [26].

\begin{tabular}{cccc}
\hline Components & F1 $(\% \mathrm{p} / \mathrm{p})$ & F2 $(\% \mathrm{p} / \mathrm{p})$ & F3 $(\% \mathrm{p} / \mathrm{p})$ \\
\hline PVA & 35 & 30.25 & 25.5 \\
HIALURONATE & 9.5 & 9.5 & 9.5 \\
SORBITOL & 27.9 & 27.9 & 27.9 \\
PEO 12 NF (1000 kDa) & 13.50 & 13.50 & 13.50 \\
SODIUM ALGINATE & 9.5 & 14.25 & 19 \\
VITAMIN E & 3.6 & 3.6 & 3.6 \\
Aloe vera & 1 & 1 & 1 \\
\hline
\end{tabular}


Table 4. Vitamin E and Aloe vera films characterization and mechanical properties [26].

\begin{tabular}{cccc}
\hline & F1 & F2 & F3 \\
\hline THICKNESS $(\mu \mathrm{m})$ & $50 \pm 0.45$ & $74.00 \pm 0.55$ & $74.00 \pm 0.54$ \\
WEIGHT $\left(\mathrm{mg} / \mathrm{m}^{2}\right)$ & $20.70 \pm 0.05$ & $20.54 \pm 0.08$ & $20.66 \pm 0.07$ \\
\% VITAMIN E $(\mathrm{p} / \mathrm{p})$ & $3.54 \pm 0.10$ & $3.55 \pm 0.10$ & $3.55 \pm 0.10$ \\
WATER CONTENT $(\%)$ & $5,36 \pm 0.04$ & $2.95 \pm 0.28$ & $1.48 \pm 0.51$ \\
E $b(\%)$ & $141.83 \pm 12.43$ & $132.78 \pm 3.81$ & $128.31 \pm 2.62$ \\
TSb $\left(\mathrm{N} / \mathrm{mm}^{2}\right)$ & $4.14 \pm 1.24$ & $4.13 \pm 1.36$ & $4.74 \pm 1.41$ \\
\hline
\end{tabular}

within 24 hours in water immersion, an important property for wound care, since exudates (inflammatory fluids) from tissue damage solubilize the film, allowing drug release.

The strength and elasticity of the film were measured by the parameters: Ts $b$ and $\varepsilon b$. The $\varepsilon b$ of the film decreased with increasing alginate content from F1 to F3, as seen in "Table 3". The most resistant and least elastic formulation was F3, with a 2: 1 ratio of alginate and hyaluronate. The F1 formulation, with a 1: 1 alginate/hyaluronate ratio, showed higher elongation capacity and lower tensile strength. This behavior may be due to the higher water content resulting from the hygroscopic contribution of hyaluronate. The intermediate formulation F2 showed a tensile strength equal to F1 and a percentage of $\varepsilon b$ not significantly different from F3.

An important factor for the properties of films is the amount of plasticizers. The addition of plasticizer reduces intermolecular forces along polymer chains, improving their flexibility, while reducing stress on disruption. These interactions are necessary to prevent films from becoming brittle, thus facilitating their handling and use, although their use implies changes in the polymer structure. In prepared films, the increase in alginate content led to lower Ts and $\varepsilon b$ values despite a constant sorbitol plasticizer content. However, this may be significantly related to the reduction of the residual water content. In fact, water molecules are generally considered to be the most natural plasticizers of hydrophilic polymer-based films.

In the study by Ammar et al. (2013), 15 different formulations were prepared with variations in component concentrations. The formulations chosen for testing were those with a total polymer concentration of $5 \%$, as it was the concentration that presented the best release efficacy [2].

The polymers used were: Eudragit EE, RL and RS and polyvinylpyrrolidone (PVP). Also, as part of the formulations were plasticizer, ethanol, and the drug (Ketorolac, anti-inflammatory), as described in "Table 5".

These different formulations were chosen with different release profiles to investigate whether they would show the same pattern in the permeation study as in the release study or not.

In the release study, in order to increase the release efficiency of ketorolac of 
Table 5. SFF formulation composition (\%p/p) [2].

\begin{tabular}{cccccccc}
\hline FORMULATION & ERS & ERL & EE & PVP & DIBUTILFTALATE & ETHANOL & DRUG \\
\hline F1 & $5 \%$ & - & - & - & $4 \%$ & $89 \%$ & $2 \%$ \\
F4 & - & $5 \%$ & - & - & $4 \%$ & $89 \%$ & $2 \%$ \\
F13 & $4 \%$ & $1 \%$ & - & - & $4 \%$ & $89 \%$ & $2 \%$ \\
F14 & $4 \%$ & - & $1 \%$ & - & $4 \%$ & $89 \%$ & $2 \%$ \\
F15 & $4 \%$ & - & - & $1 \%$ & $4 \%$ & $89 \%$ & $2 \%$ \\
\hline
\end{tabular}

the 5\% ERS (F1) film-forming polymeric solution, new formulations were prepared by combining $4 \%$ ERS with $1 \%$ more hydrophilic polymers (ERL, EE and PVP). This attempt was able to increase ketorolac release as F13-15 showed higher release efficiency when compared to $\mathrm{F} 1$. This can be attributed to the fact that the presence of hydrophilic polymers creates pores and channels that would facilitate the diffusion of drug in the films. Formulation F14 showed the highest release efficiency followed by formulation F15 and then F13. A 5\% ERL (F4) containing film-forming polymeric solution showing an intermediate sustained drug release pattern was also selected for further investigation.

Ketorolac permeation data from the pig-skin test of film-forming polymeric solutions were also studied. The five formulations showed no difference in their drug permeation profiles, although all showed different release profiles. This similarity in drug permeation profiles for F1, F4, F13, F14 and F15 may be due to the fact that the rate limitation step for ketorolac hydrophilic drug penetration through the skin is not drug release from polymeric films. But, the ability of this hydrophilic polymer to pass the barrier function of the stratum corneum.

In the literature, a good correlation between drug release and skin permeation has been described for some compounds. However, this correlation does not occur for all drugs and all drug vehicles, as shown in this study. This underlines that release experiments cannot be used to predict drug permeation through the skin and that their value in our formulation selection process is limited. Based on the physicochemical characterization, the formulation with the best properties will be modified by incorporating chemical permeation enhancers to further increase the efficiency of the delivery system. There are also patents that rely on the technology of film forming systems as components of formulations to develop their products, especially in the area of cosmetic and personal care products. In Veronique Kessler, Maximilian Angel, Klemens Mathauer's patent of BASF, 2016, the inventors made use of n-vinylpyrrolidone and modified acrylic acid hydrophobic derivative copolymers to improve the water resistance of general cosmetic formulations in addition to the ability of the copolymer increase the sun protection factor in sunscreens. Consumers search for waterproof products, with examples of eyelash masks that must be tear-resistant and sunscreen-resistant, sweat-resistant or even water-resistant for swimming outdoors. Although many products have this water resistance appeal, most have an unattractive sensory, because they are oily and sticky, and the use of film-forming 
polymers give the product a smoother sensory [27].

Hiu-Ing Donna Hwang, Grace Riccardi, inventors of the patent also published in 2016, raise the issue of multifunctional products to improve skin appearance with good water resistance, color intensity and skin retention to have a good sensory over skin. These products are difficult to formulate because they are multifunctional, and the consumer expects to find all the expected characteristics in one product and any small dissatisfaction with the sensory, color or water resistance may be reason for non-adherence to the use of the product [28].

Generally, these formulations are emulsions, the stability of which is guaranteed by surfactants, which give the skin a good sensory, but are easily re-emulsified in the presence of sweat or water, which reduces the water resistance of the formulation. In addition, any pigment/dye is easily transferred to clothing and towels. By adding a film-forming polymer, these problems are solved.

\section{Final Considerations}

The content exposed in this work addressed the state of the art of the use of polymers as film forming agents in topical formulations.

It has been argued that, as simple as these systems appear to be, there are several critical points that must be considered when designing such a formulation so that it is indeed a good system from a pharmaceutical standpoint, being truly effective for the patient. Objective that is proposed is a vehicle for topically applied drugs.

There are several polymers that can be used in this type of formulation and your choice of polymer is crucial for the desired application to be successful. Not only is the choice of polymer important, as the formulation as a whole should include volatile solvent, drug, and also consider parameters such as interactions between them, solubility of components in the solvent, final viscosity of the formulation, degree of saturation/supersaturation of the drug when the film is formed in situ, among others, so that the active ingredient can be released to the skin surface.

In addition to polymer, solvent and drug other components may be included in formulations to improve their effectiveness, such as the use of plasticizers and lipid components or permeation inducers to improve drug release from the film and its permeation through the skin layers.

In the studies presented, it was elucidated that several types of characterization are necessary to conclude that the studied system is indeed a good system for topical application. There are performed from tests of the solubility of the components in the volatile vehicle and their saturation with its evaporation, to the characterization tests of the dry films, where the influence of the components on the surface of the films, the transition temperatures and elasticity, flexibility and resistance to traction are evaluated. In addition, release tests and permeation of the drug into the skin are performed so that the study is complete and can be 
transposed to human trials for use as commercial formulations.

Finally, it can be said that film-forming systems are good bets for the treatment of dermatological diseases, transdermal drug administration, as well as being a good option for solving formulation problems in the area of personal hygiene and cosmetics. In all cases, users' adherence to the use of the product or drug increases.

\section{Acknowledgements}

Cooordenação de Aperfeiçoamento de Pessoal de Nível Superior (CAPES) Code 001, and Universidade Federal do Rio de Janeiro, UFRJ.

\section{Conflicts of Interest}

The authors declare no conflicts of interest regarding the publication of this paper.

\section{References}

[1] Souza, L.K., et al. (2013) Ureasil-Polyether Hybrid Film-Forming Materials. Colloids and Surfaces B: Biointerfaces, 101, 156-161. https://doi.org/10.1016/j.colsurfb.2012.06.009

[2] Ammar, H.O., Ghorab, M., Mahmoud, A.A., Makram, T.S. and Ghoneim, A.M. (2011) Rapid Pain Relief Using Transdermal Film Forming Polymeric Solution of Ketorolac. Pharmaceutical Development and Technology, 18, 1005-1016. https://doi.org/10.3109/10837450.2011.627867

[3] Vincent, N., Devi, D.R. and Hari, B.N.V. (2014) Progress in Psoriasis Therapy via Novel Drug Delivery Systems. Dermatology Reports, 8, 5451.

[4] Frederiksen, K., Guy, R.H. and Petersson, K. (2015) Formulation Considerations in the Design of Topical, Polymeric Film-Forming Systems for Sustained Drug Delivery to the Skin. European Journal of Pharmaceutics and Biopharmaceutics, 91, 9-15. https://doi.org/10.1016/j.ejpb.2015.01.002

[5] Garvie-Cook, H., Frederiksen, K., Petersson, K., Guy, R.H. and Gordeev, S.N. (2015) Characterization of Topical Film-Forming Systems Using Atomic Force Microscopy and Raman Microspectroscopy. Molecular Pharmaceutics, 12, 751-757. https://doi.org/10.1021/mp500582j

[6] Delambra, E. and Dimri, G.P. (2008) Cellular Senescence and Skin Aging. In: Dayan, N., Eds., Skin Aging Handbook, William Andrew Inc., Norwich, 129-148. https://doi.org/10.1016/B978-0-8155-1584-5.50011-9

[7] Kilpatrick-Liverman, L., Mattai, J., Tinsley, R. and Wu, J. (2009) Mechanisms of Skin Hydration. In: Barel, A.O., et al., Handbook of Cosmetic Science and Technology, Informa Healthcare, New York, 91-106.

[8] Kathem, K. and Kathpaliam, H. (2017) Film Forming Systems for Topical and Transdermal Drug Delivery. Asian Journal of Pharmaceutical Sciences, 12, 487-497. https://doi.org/10.1016/j.ajps.2017.07.004

[9] Marescam, V., Florim, E. and Picardom, M. (2015) Skin Phototype: A New Perspective. Pigment Cell \& Melanoma Research, 28, 378-389.

https://doi.org/10.1111/pcmr.12365

[10] Kuwazuru, O., Saothong, J. and Yoshikawa, N. (2008) Mechanical Approach to Ag- 
ing and Wrinkling of Human Facial Skin Based on the Multistage Buckling Theory. Medical Engineering \& Physics, 30, 516-522.

[11] Naylor, E.C., Watson, R.E.B. and Sherratt, M.J. (2011) Molecular Aspects of Skin Ageing. Maturitas, 69, 249-256. https://doi.org/10.1016/j.maturitas.2011.04.011

[12] Baumann, L. (2007) Skin Aging and Its Treatment. The Journal of Pathology, 211, 241-251. https://doi.org/10.1002/path.2098

[13] Garvie-Cook, H., Frederiksen, K., Petersson, K., Guy, R.H. and Gordeev, S.N. (2015) Biophysical Elucidation of the Mechanism of Enhanced Drug Release and Topical Delivery from Polymeric Film-Forming Systems. Journal of Controlled Release, 212, 103-112. https://doi.org/10.1016/j.jconrel.2015.06.015

[14] Malik, D.S., Mital, N. and Kaur, G. (2016) Topical Drug Delivery Systems: A Patent Review. Expert Opinion on Therapeutic Patents, 26, 213-228. https://doi.org/10.1517/13543776.2016.1131267

[15] Pundir, S., Badola, A. and Sharma, D. (2017) Sustained Release Matrix Technology and Recent Advance in Matrix Drug Delivery System: A Review. International Journal of Drug Research and Technology, 3, 12-20.

[16] Bhowmik, D., Gopinath, H., Kumar, B.P., Duraivel, S. and Sampath, K. (2012) Recent Advances in Novel Topical Drug Delivery System. Pharma Innovation, 1, 12-31.

[17] Pereira, G.G., Guterres, S.S., Balducci, A.G., Colombo, P. and Sonvico, F. (2014) Polymeric Films Loaded with Vitamin E and Aloe vera for Topical Application in the Treatment of Burn Wounds. BioMed Research International, 2014, Article ID: 641590. https://doi.org/10.1155/2014/641590

[18] Jaber, R., Wasbrough, M.J., Holdaway, J.A. and Edler, K.J. (2015) Interactions between Quaternary Ammonium Surfactants and Polyethylenimine at High $\mathrm{pH}$ in Film Forming Systems. Journal of Colloid and Interface Science, 449, 286-296. https://doi.org/10.1016/j.jcis.2015.01.034

[19] Ranade, S, Bajaj, A, Londhe, V., Babul, N. and Kao, D. (2017) Fabrication of Topical Metered Dose Film Forming Sprays for Pain Management. Journal of Colloid and Interface Science, 100, 132-141. https://doi.org/10.1016/j.ejps.2017.01.004

[20] Felton, L.A. (2013) Mechanisms of Polymeric Film Formation. International Journal of Pharmaceutics, 457, 423-427. https://doi.org/10.1016/j.ijpharm.2012.12.027

[21] Haider, S., Al-Masry, W., Al-Zeghayer, Y., Al-Hoshan, M. and Ali, F.A. (2011) Fabrication Chitosan Nano Fibers Membrane via Electrospinning. TechConnect Briefs, 1, 810-812.

[22] Edwards, A., Qi, S., Liu, F., Brown, M.B. and Mcauley, W.J. (2017) Rationalising Polymer Selection for Supersaturated Film Forming Systems Produced by an Aerosol Spray for the Transdermal Delivery of Methylphenidate. European Journal of Pharmaceutics and Biopharmaceutics, 114, 164-174. https://doi.org/10.1016/j.ejpb.2017.01.013

[23] Alekseev, K.V., et al. (2012) Polymers in the Formulation of Drug Dosage Forms with Modified Release. Russian Journal of General Chemistry, 82, 564-571. https://doi.org/10.1134/S1070363212030358

[24] Srivastava, A., et al. (2016) Polymers in Drug Delivery. Journal of Biosciences and Medicines, 4, 69-84. https://doi.org/10.4236/jbm.2016.41009

[25] Vijayendra, S.V.N. and Shamala, T.R. (2014) Film Forming Microbial Biopolymers for Commercial Applications-A Review. Critical Reviews in Biotechnology, 34, 338-357. https://doi.org/10.3109/07388551.2013.798254

[26] Tongnuanchan, P., Benjakul, S., Prodpran, T. and Nilsuwan, K. (2015) Emulsion 
Film Based on Fish Skin Gelatin and Palm Oil: Physical, Structural and Thermal Properties. Food Hydrocolloids, 48, 248-259.

https://doi.org/10.1016/j.foodhyd.2015.02.025

[27] Kessler, V., Angel, M. and Mathauer, K. (2016) Water-Resistant Cosmetic Formulations Comprising a Hydrophobically Modified Vinylpyrrolidone Copolymer. US Patent No. 9364418 B2.

[28] Hwang, H.I.D. and Riccardi, G. (2015) Cosmetic Compositions with Enhanced Color Retention for Improved Skin Appearance. US Patent No. 20150342845. 